\title{
Hydrothermal Wave in a Shallow Liquid Layer
}

The oscillatory thermocapillary convection and hydrothermal wave in a shallow liquid layer, where a temperature difference is applied between two parallel sidewalls, have been numerically investigated in a two-dimensional model. The oscillatory thermocapillary convection and hydrothermal wave appear if the Marangoni number is larger than a critical value. The critical phase speed and critical wave number of the hydrothermal wave agree with the ones given analytically by Smith and Davis in the microgravity environment, and it travels in the direction opposed to the surface flow. Another wave traveled downstream in addition to the hydrothermal wave traveled upstream was observed in the case of earth gravity condition.

\section{Mail Address:}

\section{Z. M. Tang, W.R. Hu}

National Microgravity Laboratory/CAS

Institute of Mechanics

Chinese Academy of Sciences

Beijing 100080, China

\section{INTRODUCTION}

The float-zone technology is a containerless process, in which the contamination and thermal stress resulted from container boundary can be removed. Onset of oscillatory convection relates to the appearance of striations in the crystal, and the thermocapillary oscillatory convections in the liquid bridges were observed in microgravity environment 1978 [1] and 1979 [2, 3]. Many attentions have been applied to study the thermocapillary convection due to the importance in both the basic research in microgravity fluid physics and the applications in space materials processing. After studying of two-dimensional and steady thermocapillary flows [4], Smith and Davis [5, 6] analyzed the instability on thermocapillary convection and found the hydrothermal waves in an infinite liquid layer, which bounded by an adiabatic bottom wall and a free surface with a constant temperature gradient. Their conclusion shown that, the gravity added in the normal stress condition can stabilize oscillation in thermocapillary convection. Villers and Platten investigated the instability in the case of coupled buoyancy and thermocapillary convection [7]. Shevtsova et al reported the stability diagram

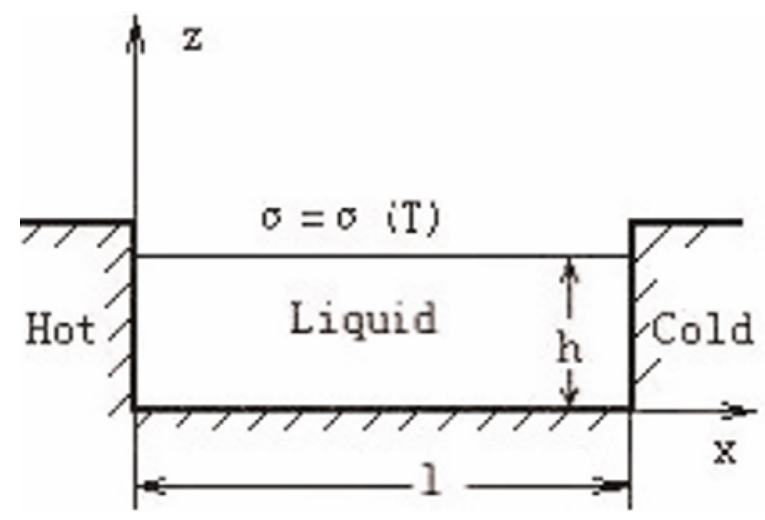

Fig. 1 A sketch of liquid layer 
for combined thermocapillary-buoyancy convection [8]. The characteristics and stabilities on 2D and 3D thermocapillary convection were studied numerically in the paper [9]. Schwabe et al investigated the thermocapillary instability of the liquid layer with Prandtl number of 17 in an annular gap, and the short-wavelength and long-wave instabilities were observed in different depth of liquid layer [10, 11].

In the present paper, we examine numerically the $2 \mathrm{D}$ thermocapillary convection and hydrothermal wave in case of large Prandtl number fluid in a shallow slot. The effect of gravity on the hydrothermal wave is also studied in detail.

\section{PHYSICAL AND MATHEMATICAL MODEL}

Consider a 2D fluid layer of silicon oil $(\mathrm{Pr}=105.6)$ in a rectangular cavity. The liquid layer is $4 \mathrm{~mm}$ in height and $52 \mathrm{~mm}$ in width. The temperature $T_{h}$ at the left side of the cavity is higher than $T_{c}$ at the right wall, and $\Delta T=T_{h}-T_{c}$. The bottom boundary is rigid, while the top free surface is a deformable and a passive gas is above. Both the bottom and top boundaries are considered as adiabatic. The liquid is considered as incompressible fluid with constant viscosity and thermal diffusivity. The equations and boundary conditions of the Boussinesq fluid are expressed by non-dimensional vorticity $\omega=\left(\omega_{\xi}, \omega_{\theta}, \omega_{\varsigma}\right)$ and stream function $\boldsymbol{\psi}=\left(\psi_{\xi}, \psi_{\theta}, \psi_{\varsigma}\right)$ as follows.

$$
\begin{aligned}
& \nabla \times \nabla \times \boldsymbol{\psi}=\boldsymbol{\omega} \\
& \frac{\partial \boldsymbol{\omega}}{\partial t}+V \cdot \nabla \boldsymbol{\omega}-\boldsymbol{\omega} \cdot \nabla \mathbf{V}=\frac{1}{R e}\left[\nabla^{2} \boldsymbol{\omega}+\frac{G r}{R e} \nabla \times(T e)\right] \\
& \frac{\partial \Theta}{\partial t}+\boldsymbol{V} \cdot \nabla \Theta=\frac{1}{M a} \nabla^{2} \Theta .
\end{aligned}
$$

where $\boldsymbol{V}=(U, 0, W)$ is non-dimensional velocity, $\boldsymbol{e}$ is the unit vector of acceleration and only the gravity is considered here, non-dimensional temperature $\Theta=\left(T-T_{c}\right) /\left(T_{h}-T_{c}\right)$, Non-dimensional parameters

$$
G r=\frac{g \beta \Delta T \ell^{3}}{v^{2}}, R e=\frac{|\partial \sigma / \partial T|^{T \ell}}{\rho v^{2}}
$$

and $M a=R e P r$ are respectively the Grashof number, Reynolds number and Marangoni number, where $P r=v / \kappa$ is Prandtl number. In the case of two-dimensional problem, only $\psi_{\theta}$ and $\omega_{\theta}$ are not zero, which are pointed by $\psi$ and $\omega$ in the following text. The boundary conditions are as follows:

$$
\begin{aligned}
& \xi=0: \quad \psi=0, \quad \omega=-\frac{\partial w}{\partial \xi} \quad \Theta(0, \tau)=f(\tau), \\
& \xi=1: \quad \psi=0, \quad \omega=-\frac{\partial w}{\partial \xi} \quad \Theta(1)=0, \\
& \varsigma=0: \quad \psi=0, \quad \omega=-\frac{\partial u}{\partial \varsigma} \quad \frac{\partial \Theta}{\partial n}=0, \\
& \varsigma=R(\xi, \tau) 0: \quad \psi=0, \\
& \omega=\frac{\left(1+R^{\prime 2}\right) \partial T}{\left(1-R^{\prime 2}\right) \partial S}+\frac{2 R^{\prime}}{\left(1-R^{\prime 2}\right)}\left[\frac{\partial}{\partial \xi}\left(-\frac{\partial \psi}{\partial \varsigma}\right)-\frac{\partial}{\partial \varsigma}\left(\frac{1}{\xi} \frac{\partial \xi \psi}{\partial \xi}\right)\right. \\
& +2 \frac{\partial}{\partial \varsigma}\left(-\frac{\partial \psi}{\partial \varsigma}\right) \\
& \frac{\partial \Theta}{\partial n}=0 \\
& \left(P-P_{\infty}\right)=\frac{2}{\left(1+R^{\prime 2}\right)}\left[R^{\prime 2} \frac{\partial U}{\partial \xi}-R^{\prime}\left(\frac{\partial W}{\partial \xi}+\frac{\partial U}{\partial \varsigma}\right)+\frac{\partial W}{\partial \varsigma}\right] \\
& -\frac{R^{\prime \prime}}{C a \cdot\left(1+R^{\prime 2}\right)^{3 / 2}} \text {. }
\end{aligned}
$$

\begin{tabular}{|c|c|c|c|c|c|c|c|c|c|}
\hline $\operatorname{Pr}=1$ & Grid & $R e$ & $-\psi_{\min }$ & $\omega_{\text {core }}$ & $x_{c}$ & $z_{c}$ & $u(0.5,1)$ & $N u_{x=0}$ & $N u_{x=1}$ \\
\hline $\mathrm{A}$ & $64 \times 64$ & $1.0 \times 10^{4}$ & $3.23 \times 10^{-3}$ & $-6.97 \times 10^{-2}$ & 0.58 & 0.62 & $2.96 \times 10^{-2}$ & 4.36 & 4.40 \\
\hline $\mathrm{B}$ & $64 \times 64$ & $1.0 \times 10^{4}$ & $3.20 \times 10^{-3}$ & $-7.10 \times 10^{-2}$ & 0.58 & 0.63 & $2.95 \times 10^{-2}$ & 4.34 & 4.32 \\
\hline \multicolumn{10}{|c|}{$\operatorname{Pr}=10$} \\
\hline A & $64 \times 64$ & $1.0 \times 10^{3}$ & $2.60 \times 10^{-3}$ & $-10.0 \times 10^{-2}$ & --- & ---- & $2.30 \times 10^{-2}$ & 3.79 & 3.83 \\
\hline B & $64 \times 64$ & $1.0 \times 10^{3}$ & $2.75 \times 10^{-3}$ & $-9.56 \times 10^{-2}$ & ---- & ---- & $2.47 \times 10^{-2}$ & 3.97 & 3.89 \\
\hline
\end{tabular}

The free surface is described as $\varsigma=h / l=R(\xi)$ and $R(0)=R(l)$

Table 1. The comparison of Bradley's results [12] with present one: (A-Bradley's results, $B$ - present results) 
$=1$. Here, $\boldsymbol{n}$ and $\boldsymbol{s}$ are respectively the unit vectors in the normal and tangential direction of the free surface. The initial conditions are given by the static case with a zero applied temperature difference.

\section{NUMERICAL METHOD}

\section{AND VALIDATION OF NUMERICAL CODE}

We solved the equations (1-3) with the initial and boundary conditions by using the hybrid finite element method of fractional steps. The numbers of the cell are $121 \times 21$ in the directions $x, z$ respectively, and the calculated domain was divided in to 4800 triangular elements with 2541 nodes. The initial free surface is adopted as a plane at $t=0$. The temperature at the right wall is taken as $0^{\circ} \mathrm{C}$ for simplification. The temperature difference is gradually increased, with a heating rate $0.5^{\circ} \mathrm{C} / \mathrm{sec}$, to a determined value, then the temperature difference keeps unchangeable. The flow field and temperature distribution in the liquid layer can be then obtained numerically. The configuration of free surface is calculated by using condition (10). In comparison the present program with that obtained by Bradley

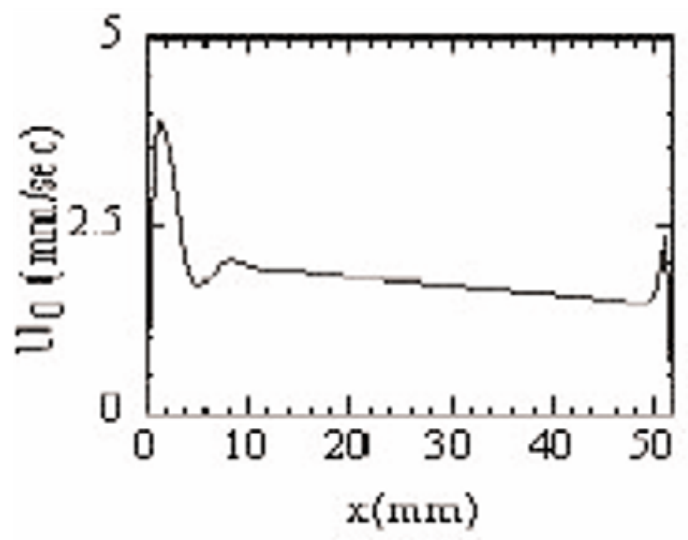

Fig. 2: The steady velocity at the free surface of the liquid layer $(g=0$, $\left.\Delta T=25^{\circ} \mathrm{C}\right)$

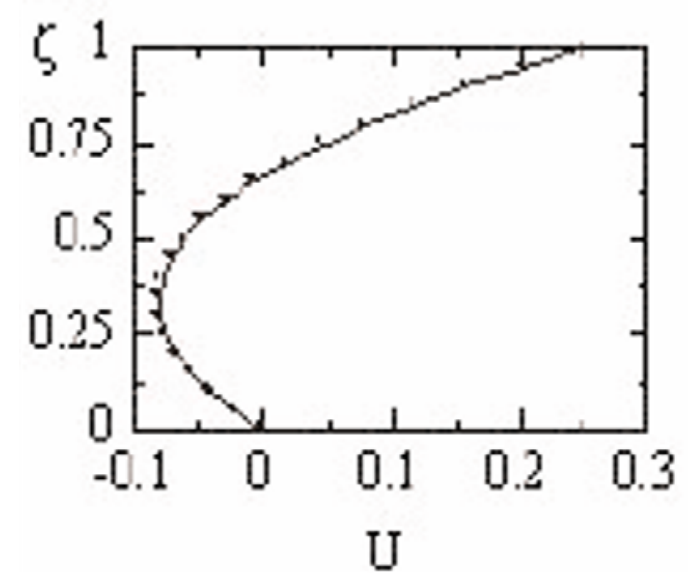

Fig. 3 The non-dimensional velocity $U$ distribution along the height $\zeta$ at $\xi=0.5\left(g=0, \Delta T=25^{\circ} \mathrm{C}\right)(-$ present result,$\cdot$ the result of Smith and Davis) et al in the case of the thermocapillary convection in a square container, the results are shown in table 1 where, $-\psi_{\min }$ is the minimum of stream function in the region, and $\omega_{\text {core }}$ is the vorticity at point $\left(x_{c}, z_{c}\right)$. The velocity at $x=0.5$ and $z=1$ is pointed by $u(0.5,1)$. Nusselt number is defined as

$$
N u(x)=\int_{0}^{1}\left(M a \frac{\partial \psi}{\partial z} T-\frac{\partial T}{\partial x}\right) d z .
$$

The results obtained by the hybrid finite element method of fractional steps are coincident with those given by Bradley, and the present calculation method and program are valid.

\section{NUMERICAL RESULTS}

\section{1 Results for $g=0$}

The time-dependent velocity, temperature and the height of the free surface are divided in to two parts as $u=u_{0}(x, z)+u^{\prime}(x, z$, $t$ ), where $u_{0}$ is a time-average and steady velocity. The oscilla-

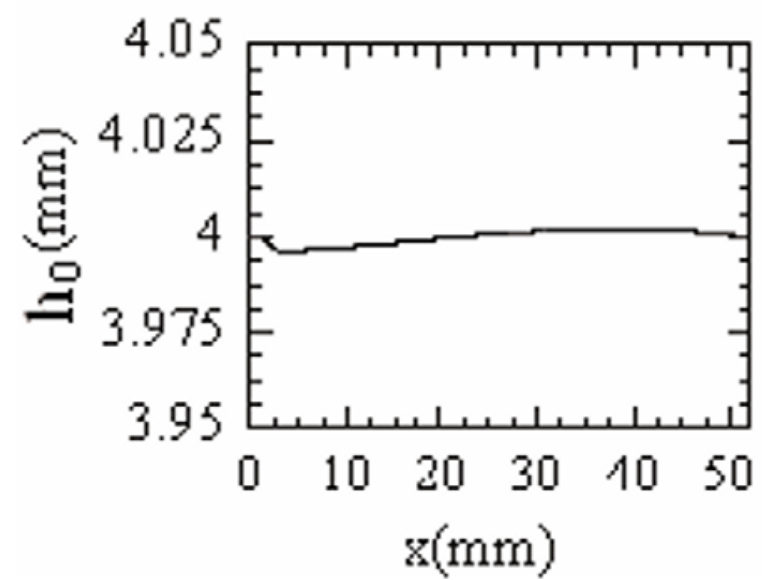

Fig. 4 The steady shape of the free surface $\left(g=0, \Delta T=25^{\circ} \mathrm{C}\right)$

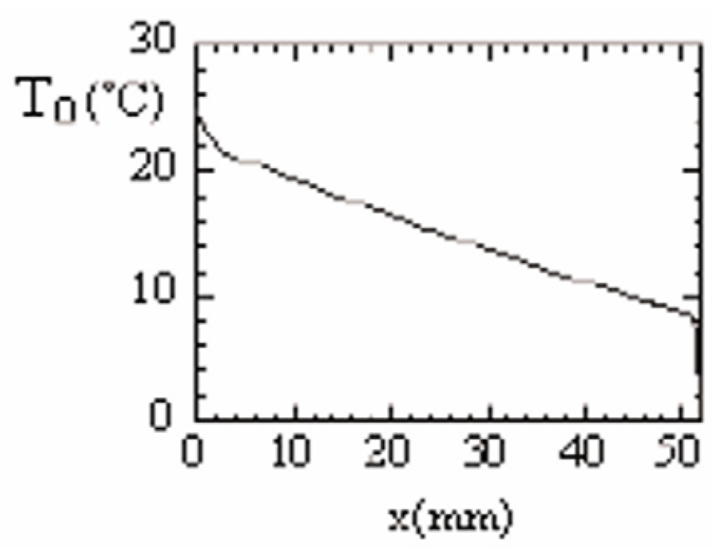

Fig. 5 The steady temperature distribution at the free surface $(g=0, \Delta T$ $=25^{\circ} \mathrm{C}$ ) 
tory component $u$ ' can be obtained from $u-u_{0}$. When $\Delta T$ equals $9^{\circ} \mathrm{C}$ the oscillatory flow and traveling wave appear in the middle region of the liquid layer, and then expends to the whole liquid layer at about $\Delta T=25^{\circ} \mathrm{C}$. The results at $\Delta T=25^{\circ} \mathrm{C}$ will be consider as the critical state. Distribution of steady velocity $u_{0}$

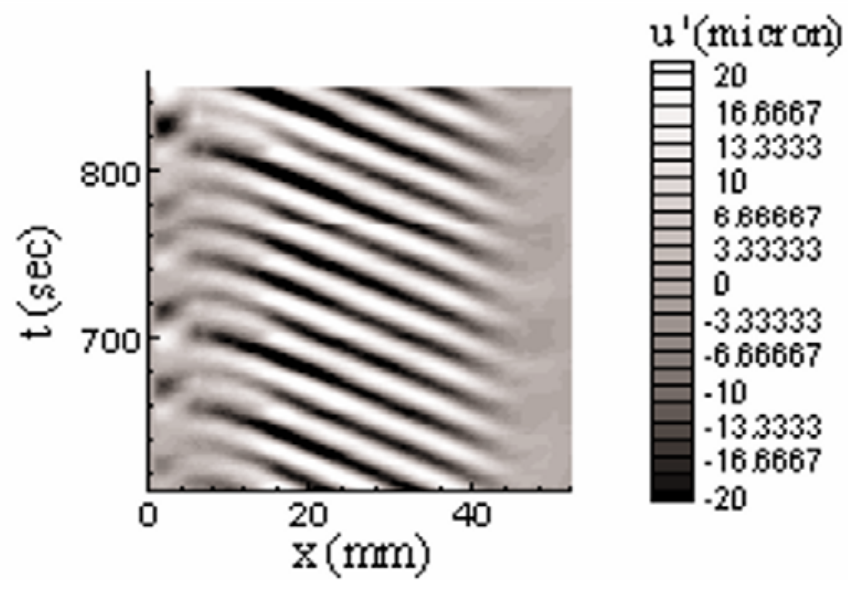

Fig. 6 The evolution of velocity u' at the free surface $(g=0, . T=25 \circ C)$

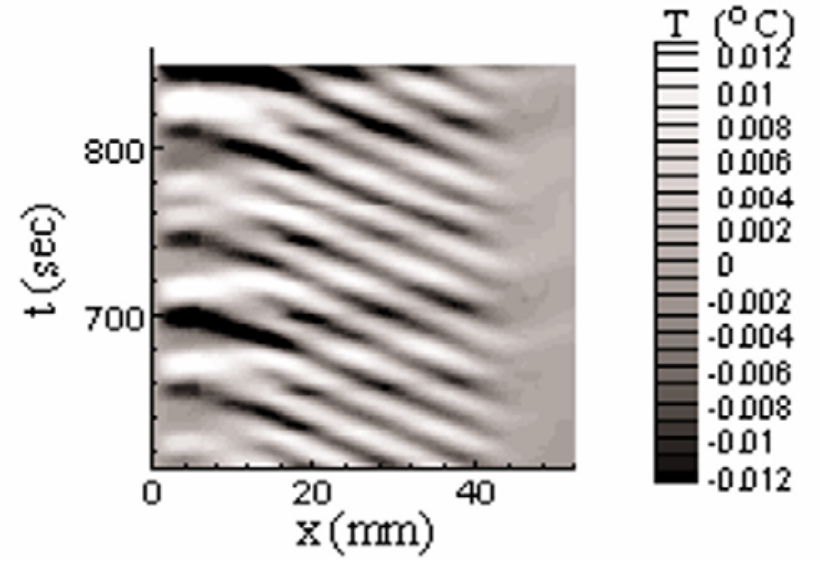

Fig.7 The evolution of temperature $T$ ' at the free surface $(g=0, \Delta T=$ $25^{\circ} \mathrm{C}$ )

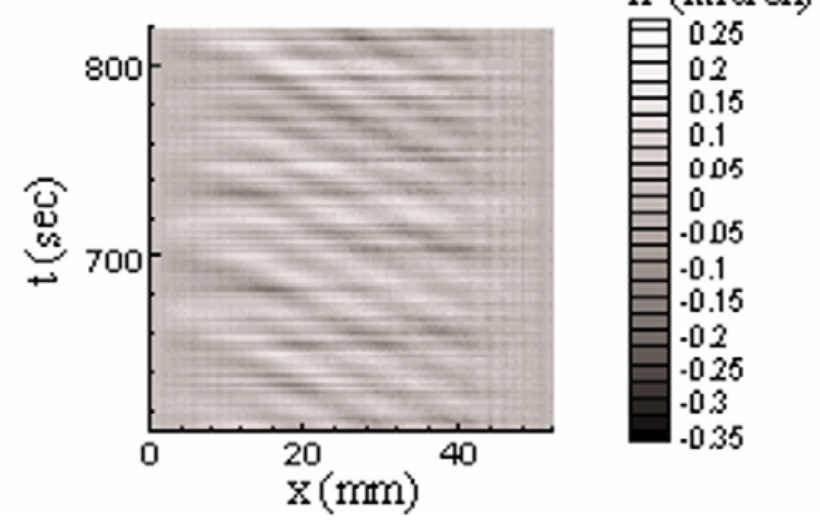

Fig. 8 The time-dependent height $h$ ' of free surface $\left(g=0, \Delta T=25^{\circ} \mathrm{C}\right)$ at the free surface is shown in figure 2 . The velocities are larger in the regions near the left and the right walls. The distribution of non-dimensional velocity $U$ along the height $\varsigma$ at $\xi=0.5$ quite agree with the basic solution of Smith and Davis [5] as shown in Fig.3, where the solid line was the computational results and the dark dots were given by Smith and Davis. The configuration of the free surface and the temperature distribution along the free surface are shown respectively in figures 4 and 5. The temperature decreases linearly in the central region of the free surface. The oscillatory components of velocity, temperature and the height of the free surface are given respectively in Fig.6, Fig.7 and Fig.8, the figures show the travelingwave propagating in the opposite direction of the velocity at free surface and was named as upstream traveling wave. The oscillatory components $u$ ' are shown at three instants in Fig. 9, it relates to the wavelength of $10.39 \mathrm{~mm}$. The temperature decreases linearly and the wave is regular in the central region, where has less influence by the sidewalls. Therefore, the temperature gradient and wavelength are determined from the results in the central region from $x=15 \mathrm{~mm}$ to $x=40 \mathrm{~mm}$. The data are organized similar to the form as Smith and Davis [5] for comparison. Non-dimensional wavenumber and phase velocity in the present paper are respectively 2.417 and 0.0666 , which

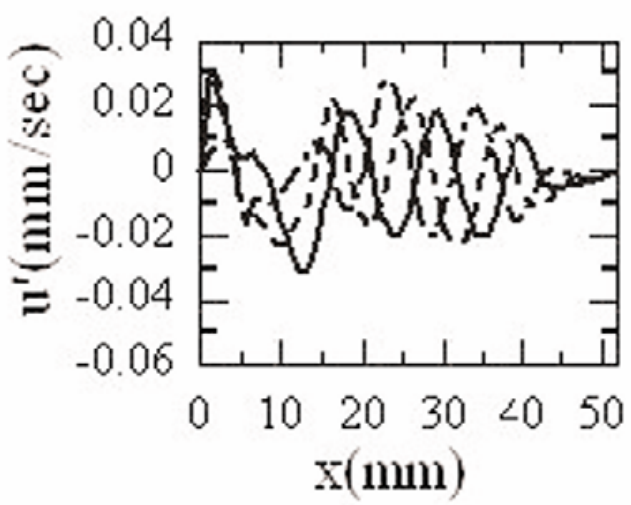

Fig. 9 Oscillatory velocity $u$ ' at the free surface $\left(g=0, \Delta T=25^{\circ} \mathrm{C}\right)$

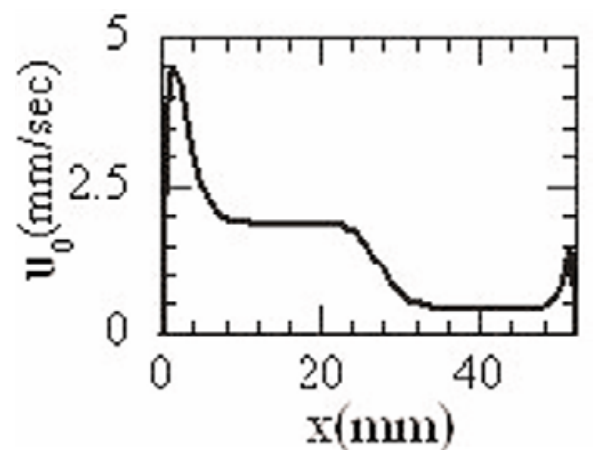

Fig 10 The steady velocity $u_{0}$ at Free surface $\left(g=g_{e^{\prime}} \Delta T=15^{\circ} \mathrm{C}\right)$ 
are close to the critical wave number 2.47 and critical phase velocity 0.0622 given by Smith and Davis for $\operatorname{Pr} \rightarrow \infty$.

\subsection{Results for $g=g_{e}$}

Usually, the effect of gravity is considered in the normal-stress boundary condition, and the stabilization effect of gravity on the interface of liquid layer has been suggested [5]. The buoyancy effect is considered in this section. The steady velocity components $u_{0}$ and the temperature $T_{0}$ at the free surface are shown in Fig. 10 and Fig. 11 respectively. Figs. 12 and 13 correspond to the traveling wave of $u$ ', and $T$ ". There are two traveling waves in each figure, one of them propagates upstream and another one downstream. Both of them have the same oscillatory frequency. The wavelength of the upstream wave is obviously smaller than that of the downstream wave. In these figures the traveling waves are observed in the region near the hotter left wall. The calculations were conducted at $\Delta T=25^{\circ} \mathrm{C}$ for $g=g_{\mathrm{e}}$, and the data of the upstream hydrothermal wave are organized according to the form in paper [5]. When $g=g e$. Owing to the effect of buoyancy the stronger convection exists in the liquid layer, therefore the temperature gradient $\partial T / \partial x$, Reynolds number Re and Marangoni numbers $M a$ are larger than that for $g=$ 0 . Comparing with the results of $g=g_{0}$, the phase velocity of the hydrothermal wave is smaller due to the larger wavenumber although the frequency is larger for $g=g_{\mathrm{e}}$.

\section{CONCLUSIONS AND DISCUSSION}

If the free surface is considered as a plane, all results are the same as that with changeable free surface. It means that the

\begin{tabular}{ccccccc}
\hline $\mathbf{g}$ & $\partial \mathrm{T} / \partial \mathbf{x}$ & $\boldsymbol{R e}$ & $\mathbf{M a}$ & $\mathbf{f}(1 / \mathbf{s e c})$ & $\kappa$ & $\mathbf{c}$ \\
\hline 0 & 2.74 & 2.81 & 297 & 0.04508 & 2.417 & 0.0666 \\
\hline$g_{e}$ & 3.10 & 3.18 & 336 & 0.04782 & 3.131 & 0.0482 \\
\hline
\end{tabular}

Table 2. The comparison of upstream hydrothermal wave at $g=0$ with that at $g=g_{e}, \Delta T=25^{\circ} \mathrm{C}$

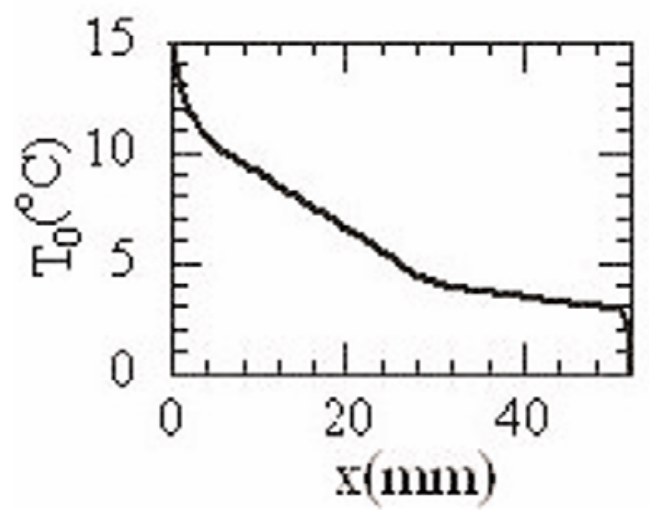

Fig.11 The steady temperature $T_{0}$ at free surface $\left(g=g_{e}, \Delta T=15^{\circ} \mathrm{C}\right)$ deformation of the free surface is not the reason of exciting the traveling wave, and the oscillation of the free surface is only the response to oscillation of flow and temperature in the liquid layer. The steady maximum deformation of the free surface at $g$ $=0$ and $\Delta T=25^{\circ} \mathrm{C}$ is 4.18 micron meter, about $0.1 \%$ of the height of the liquid layer, and the amplitude of $h$ ' is only about 0.1 micron.

The wavenumber and phase velocity of the two-dimensional, upstream traveling wave, obtained at the present paper for $g=$ $0, \Delta T=25^{\circ} \mathrm{C}$, are close to the values presented by Smith and Davis [5]. But the Marangoni number 297 is less than critical value 398.5 evaluated as $P r \rightarrow \infty$.in paper [5].

In the present calculation the Bond number $B o=\rho g h^{2} / \sigma=$ 7.29 , the gravity effect is important. The effects of gravity on the thermocapillary convection of liquid layer are included in the following three aspects.

(1) The surface velocity in the wave region is about 4 times larger than that in the region near the cold wall as shown in Figure 10. The temperature decreases linearly in the left region of figure 11. These results are different from that for $g=0$.

(2) Two traveling waves are observed for gravity $g=g_{e}$, the upstream hydrothermal wave is superimposed on the downstream long wave. The downstream long wave originates at the

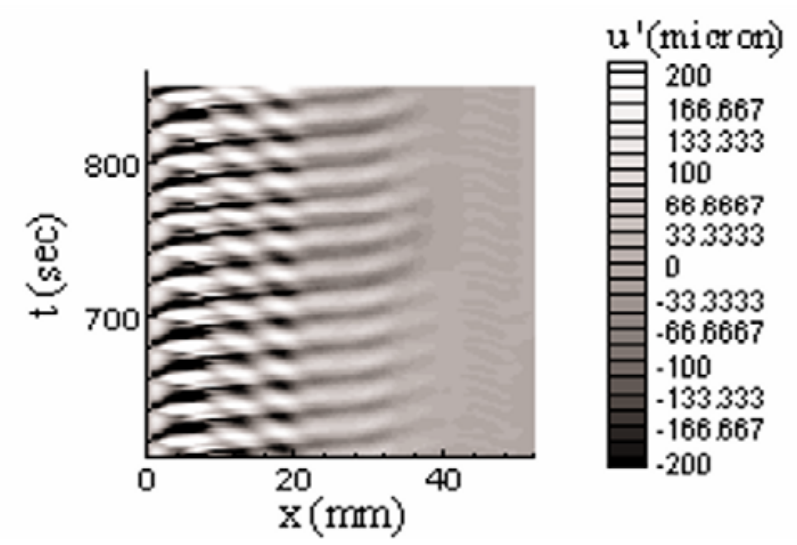

Fig. 12 The evolution of velocity $u^{\prime}$ at the free surface $\left(g=g_{e^{\prime}} \Delta T=\right.$ $\left.15^{\circ} \mathrm{C}\right)$

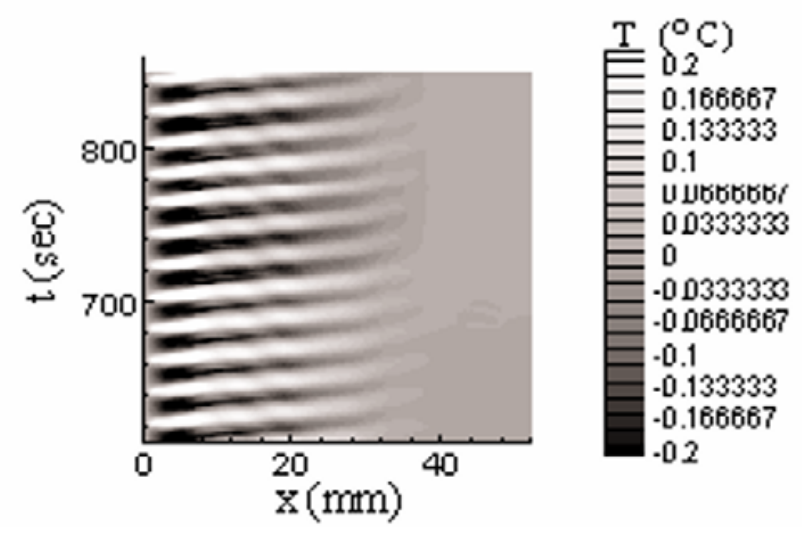

Fig.13 The evolution of temperature T' at the free surface $\left(g=g_{e^{\prime}} \Delta T\right.$ $=15^{\circ} \mathrm{C}$ ) 
place near the left hot wall.

(3) The amplitudes of oscillatory velocity $u$ ' and oscillatory temperature $T^{\prime}$ are about one order larger on the ground than those in microgravity environment.

Smith and Davis found the long-wave instability in the case of shear flow in small Reynolds number [13]. They pointed that the unstable long-wave disturbance always travel in the direction of decreasing pressure, which agrees with the computational results of figures 12 and 13 . We also notice the preferred long-wave mode was observed at $h>1.4 \mathrm{~mm}$ in Schneider's experiment [10].

The results for $g=g_{e}$ will be compared with that of the experiment on the ground. It is interesting to study the effects of parameters on hydrothermal wave, for example the length $l$, the depth $h$ of liquid layer, $\operatorname{Pr}$ number and gravity level. The bifurcation process of hydrothermal wave as increasing $M a$ number will be discussed in the future.

\section{ACKNOWLDEGMENT}

The project is supported partly by the National Natural Science Foundation (10432060) of China.

\section{REFERENCES}

1. Schwabe D., Scharmann A., Preisser F. and Oeder R., Experiments on surface tension driven flow in floating zone melting, J. of Crystal Growth, Vol. 43, p.305, (1978)

2. Chun Ch. H. and Wuest W., Experiments on the transition from the steady to oscillatory Marangoni convection of a floating zone under reduced gravity effect, Acta Astronautica, Vol. 6, p.1073, (1979)

3. Schwabe D. and Scharmann A., Some evidence for the existence and magnitude of a critical Marangoni number for the onset of oscillatory flow in crystal growth melts, J. of Crystal Growth, Vol. 46, p.125, (1979)

4. Sen A. K. and Davis S. H., Steady thermocapillary flows in two-dimensional slots, J. Fluid Mech., Vol. 121, p.163, (1982)

5. Smith M. K. and Davis S. H., Instability of dynamic thermocapillary liquid layer, Part 1. convective instability, J. Fluid Mech., Vol. 132, p.119, (1983)

6. Smith M. K. and Davis S. H., Instability of dynamic thermocapillary liquid layer, Part 2. Surface-wave instabilities, J. Fluid Mech., Vol. 132, p.145, (1983)

7. Villers D. and Platten J. K., Coupled buoyancy and Marangoni convection in acetone: experiments and comparison with numerical simulation, J. Fluid Mech., Vol. 234, p.487, (1992)

8. Shevtsova V.M., Nepomnyashchy A. A. and Legros J. C., Thermocapillary - buoyancy convection in a shallow cavity heated from the side. Physical Review E 67, 066308 (2003)

9. Xu J. and Zebib A., Oscillatory two- and three-dimensional thermocapillary convection. J. Fluid Mech., Vol. 364, p.187, (1998)

10. Schneider J., Schwabe D. and Scharmann A., Experiments on surface waves in dynamic thermocapillary liquid layers, Microgravity Sci. Technol.Vol. IX/2, p.86, (1996)

11. Schwabe D., Moller U., Schneider J. and Scharmann A., Instabilities of shallow dynamic thermocapillary liquid layers, Phys. Fluids A, Vol. 4 (11) p.2368, (1992)

12. Carpenter Bradley M. and Homsy G. M., High Marangoni number convection in a square cavity part II, Phys. Fluids A, Vol. 2(2), p.137, (1990)

13. Smith M. K. and Davis S. H., The instability of sheared liquid layers, J. Fluid Mech. Vol. 121, p.187, (1982) 